Y. C. Minh and E. F. van Dishoeck, eds.

\title{
New Developments in Inner Solar Nebula Chemistry
}

\author{
Monika E. Kress \\ Mail Stop 245-3, Space Science Division, NASA Ames Research Center, \\ Moffett Field, California 94035-1000, USA
}

\begin{abstract}
The molecular cloud material which became the asteroids, meteorites and inner planets was extensively processed in the inner solar nebula. The chemical complexity of the most ancient meteorites attests to the highly nonequilibrium nature of the chemistry in this region, particularly regarding the volatile elements. Theoretical models describing the time evolution of the temperature and density profiles of protoplanetary accretion disks have recently become available. Such models provide a realistic framework within which to study, for instance, the effects of lightning and surface-catalyzed (gas-grain) reactions in the inner nebula. Here, we show that the latter would have been most efficient during the meteorite-forming epoch of the nebula, at the present position of the asteroid belt.
\end{abstract}

\section{Introduction and Previous Work}

Inner solar nebula chemistry is the study of the final stages of chemical evolution from molecular clouds to planetary systems. Mercury, Venus, Earth, Mars, and the asteroids and meteorites formed in the inner solar nebula. What is known about the conditions and processes there has largely been based on studies of unequilibrated chondritic meteorites, the least-altered material left over from this epoch. It has recently become possible to study the solar nebula from an astronomical perspective. The gas-rich protoplanetary disks around $\mathrm{T}$ Tauri stars such as DM Tau and GG Tau are believed to be representative of the solar nebula. Organic molecules have been observed in these objects and in the circumstellar disks around Herbig Ae stars (Dutrey et al. 1997, 2000; Qi et al. 1999; Thi et al. 1999; Velusamy et al. 1999). While direct observations of the terrestrial-planet-forming regions of disks are not yet possible, several groups have developed theoretical models which can be used to infer the physical conditions there (e.g. Boss 1993; Cassen 1996; Bell et al. 1997, 2000; Stepinski 1998; Woolum \& Cassen 1999). The unique challenge for the study of inner solar nebula chemistry is that the problems are constrained at one extreme by very large distant objects (protoplanetary disks), and at the other extreme by chondritic meteorites, the remnants of the inner solar nebula that we can hold in our hands.

Fegley and co-workers pioneered the study of solar nebula chemistry (see reviews by Fegley 1993, Prinn \& Fegley 1989, and references therein) using thermochemical equilibrium models with adiabatic pressure-temperature profiles 
(e.g. Lewis 1974). They find that, if the solar nebula had been in equilibrium, $\mathrm{C}$ and $\mathrm{N}$ would have been in the form of $\mathrm{CO}$ and $\mathrm{N}_{2}$ in the inner nebula, and $\mathrm{CH}_{4}$ and $\mathrm{NH}_{3}$ in the outer. Thus, Fegley (1993) emphasizes the importance of thermochemical models in identifying departures from equilibrium (such as the existence of organics in meteorites and CO in comets) and the need for chemical kinetic models to quantitatively explain them.

Aikawa et al. (1997) and Willacy et al. (1998) present time-dependent nonequilibrium chemical models for protoplanetary disks. Aikawa et al. (1997) show that the highly nonequilibrium combination of $\mathrm{CO}_{2}$ and $\mathrm{NH}_{3}$ ice, which appears to exist in some comets, can form via ion-molecule chemistry driven by cosmic rays in the outer nebula. Willacy et al. (1998) model the chemistry throughout the disk, extending well into the inner nebula. They include ionization by cosmic rays and decay of ${ }^{26} \mathrm{Al}$, and find that the chemistry in the inner disk is nevertheless dominated by neutral-neutral reactions, and also that relatively high abundances of nonequilibrium species can exist there. Finocchi \& Gail (1997) study the effects of ionization due to radionuclides ${ }^{26} \mathrm{Al}$ and ${ }^{60} \mathrm{Fe}$. They conclude that these energetic processes do indeed drive ion-molecule reactions that form molecules which could not derive from neutral chemistry alone. Igea \& Glassgold (1999) calculate the ionization rate from stellar X-rays in the disks around T Tauri stars. They find that the ionization rate is a function of vertical column density into the disk. Column densities are very high at the midplane of the inner solar nebula $\left(\sim 10^{27} \mathrm{H}\right.$ atoms $\left.\mathrm{cm}^{-2}\right)$, which may preclude stellar $\mathrm{X}$-rays from directly participating in the chemistry there.

Duschl et al. (1996) present a model for dust destruction processes in the inner regions of protoplanetary accretion disks. They find that there is a delicate interrelationship between the dust composition and temperature structure of the disk, since the dust destruction processes are a sensitive function of temperature, while dust composition determines the opacity and hence the temperature. Gail (1998) models the composition and structure of the refractory dust component consisting of $\mathrm{Si}, \mathrm{Fe}, \mathrm{Mg}, \mathrm{Ca}$, and $\mathrm{Al}$ using chemical equilibrium models. Finocchi et al. (1997) find that the destruction of carbonaceous dust grains via oxidation by $\mathrm{OH}$ radicals is more efficient than vaporization (e.g. thermal destruction). $\mathrm{OH}$ radicals convert the carbon-based dust into gas-phase hydrocarbons; this step is rapid compared to that which converts these hydrocarbons into $\mathrm{CO}$, allowing large amounts of $\mathrm{CH}_{4}$ and other organics to persist for some time in the inner disk (within $2 \mathrm{AU}$ ).

Three recent developments in inner solar nebula studies are highlighted in this paper. First, lightning has been shown to be a potentially important process in the inner nebula (Desch \& Cuzzi 2000), and its possible consequences on the chemistry are discussed. Next, theoretical models for the solar nebula and protoplanetary disks are now available, providing a more realistic framework within which to study nebular chemistry. Finally, grain-surface catalyzed CO dissociation and hydrogenation has been modeled in detail (Kress \& Tielens 1999). This model is applied to inner solar nebula conditions calculated using the theoretical disk models of Bell et al. (1997). We show that, in the presence of iron dust grains, surface catalysis would have occurred in the nebula in the vicinity of the modern-day asteroid belt, during the epoch of the solar nebula in which the meteorites formed. 


\section{Lightning in the Solar Nebula}

Lightning has been proposed as a mechanism to melt chondrules (Horanyi et al. 1995), millimeter-sized silicate inclusions that constitute a significant fraction of a chondritic meteorite's mass and volume. While several chondrule formation mechanisms have been suggested in addition to lightning (for a detailed review of these, see Boss 1996), chondrule properties suggest that their melting mechanism must have been rapid and repeatable. Therefore, lightning is a promising candidate (Boss 1996), but a mechanism to generate it has been elusive. Desch \& Cuzzi (1999) present a model by which lightning is generated via the triboelectric charging of dust grains and chondrule precursors and size sorting of chondrules via turbulent concentration. Triboelectric charging occurs when materials of two different contact potentials (in this case, silicate chondrule precursors and small metallic iron grains) come into contact with one another. The chondrule precursors acquire a net positive charge and are concentrated by turbulence. The resulting charge separation generates electric fields, which, once large enough, will ionize the gas $\left(\mathrm{H}_{2}\right)$. The individual lightning discharges can release electrical energies of $\sim 10^{16}$ erg. The superheated channel of gas expands to reach pressure equilibrium with the surrounding nebula in $\lesssim 1 \mathrm{sec}$, and cools to nebular temperatures within about an hour (Desch \& Cuzzi 1999). The energy generated by the lightning bolt is sufficient to vaporize fine dust while only melting the chondrules (Desch, private communication).

Lightning has interesting implications for solar nebula chemistry. Prinn \& Fegley (1989) and Fegley (1993) suggested that lightning could disequilibrate gas in the outer solar nebula and giant planet subnebulae. Because Desch \& Cuzzi's mechanism for generating lightning requires a difference in contact potentials between two grain types, lightning will not be generated in this way in regions that are cold enough that grains would be coated with ice. Also, the charge separation required to establish the large electric fields which generate lightning will not occur where the ionization (e.g. via cosmic rays) is high enough to allow charge leakage. We therefore expect that, if lightning were generated via this mechanism, it will only occur in the inner solar nebula, and in addition to melting the chondrules, it will have consequences for both the nebular gas and the matrix of chondritic meteorites.

The chemistry resulting from lightning discharges is much like shock chemistry and has been studied in planetary atmospheres (e.g. Podolak \& Bar-Nun 1988). Chemical reactions in the channel of super-heated gas are very rapid, and chemical equilibrium is maintained until the gas cools below the "freeze-out" temperature $(\sim 2000 \mathrm{~K})$, below which the reaction rates are very long compared to the rate at which the gas cools (McKay \& Borucki 1997). Experimental lightning simulations (laser-induced plasmas) show that in a mixture of $\mathrm{H}_{2}$ and $\mathrm{CH}_{4}$, a variety of organics is produced as a result of the lightning discharge. However, in an $\mathrm{H}_{2} / \mathrm{CO}_{2}$ gas, no organics are formed because all of the carbon becomes locked in $\mathrm{CO}$, one of the first molecular species to form in the cooling gas (McKay \& Borucki 1997). In a lightning discharge, as in many astrophysical regions, the total $\mathrm{C} / \mathrm{O}$ ratio of the gas is very important in determining the products of lightning chemistry. In planetary atmospheres, the rate at which trace gases are produced by lightning is proportional to the rate of energy dissipated by electrical discharges (Borucki \& McKay 1987). Thus, in order to 
quantify lightning's effect on nebular gas and dust, the total energy released per discharge and the rate at which these discharges occur must be determined as a function of position in the nebula.

\section{Solar Nebula Models}

Physical models for protoplanetary disks, including the solar nebula, have been developed over the past several years by a number of groups. Such models can be used to calculate the temperature and density as a function of position within the disk. Since the chemistry of the inner disk depends crucially on the temperature, these models provide an essential framework within which to study the chemical processes that might occur there. Some models even consider the temporal evolution of these profiles. Four recent models are highlighted in this section as particularly useful in the study of nebular chemistry.

Cassen (1996) presents a relatively simple, time-dependent model for the solar nebula with parameters constrained both by meteoritic data as well as by observations of $\mathrm{T}$ Tauri star-disk systems. The model parameters are the total angular momentum and initial mass of the disk, the initial accretion rate onto the Sun, an algebraic decay index, and a characteristic timescale for the coagulation of solids at 1 AU. Stepinski (1998) emphasizes that the solar nebula should be treated as a process rather than as an object and uses time-dependent accretion disk theory to analytically model the spatial structure and time evolution of the solar nebula. These models have three free parameters: initial mass and angular momentum of the nebula, and a viscosity parameter $\alpha$. Boss (1993) uses a 2-D radiative hydrodynamics code to model protoplanetary accretion disks. Although these models are not explicitly time dependent, the model parameters (such as accretion rate and disk mass) provide "snapshots" of the nebula during various stages of evolution.

Bell et al. (1997) model the structure and appearance of viscously heated protoplanetary accretion disks. They derive analytic formulae for the radial profiles of the disk midplane temperature, opacity, pressure and surface density. These models also have three free parameters : accretion rate (mass flux, $\dot{M})$, stellar mass, and viscous efficiency $(\alpha)$. Bell et al. (2000) show that early in the solar nebula's lifetime when the mass flux is high, the inner nebula is too hot to allow the condensation or agglomeration of solids. Not until later (once $\dot{M} \lesssim 10^{-6} \mathrm{M}_{\odot} /$ year) is the inner nebula cool enough to allow silicates to condense. The midplane temperatures for the inner solar nebula derived by Bell et al. (1997) are shown in Figure 1. The two curves in each panel of Figure 1 represent the upper (for $\alpha=10^{-4}$ ) and lower (for $\alpha=10^{-2}$ ) limits of the disk midplane temperature. The range of these plots is from $400 \mathrm{~K}$ (the meteorite agglomeration temperature) to $1400 \mathrm{~K}$ (the vaporization temperature of silicates). Meteorites can only form in this temperature range, indicating that meteorite formation could have begun when $\dot{M} \sim 10^{-6} \mathrm{M}_{\odot} /$ year and must have been essentially complete by the time $\dot{M} \sim 10^{-8} \mathrm{M}_{\odot} /$ year.

Also shown in Figure 1 are the locations of the inner planets and three families of asteroids. There is some consensus in the asteroid/meteoritics community that the organic-rich carbonaceous chondrites originate from the C-type asteroids (Wetherill \& Chapman 1988). However, it is currently a subject of great 
controversy whether the ordinary chondrites are related to the S-type asteroids (Meibom \& Clark 1999; Bell et al. 1989). Dynamical arguments suggest that it is unlikely that there are any meteorites which derive from the Trojan asteroid family (Wetherill \& Chapman 1988). There is great motivation to identify the sources of the meteorites and to determine the extent to which they were processed after their formation. Detailed models for solar nebula chemistry are essential in order to elucidate these issues.

\section{CO Hydrogenation on FeNi Grains: A Case Study of Gas-Grain Chemistry in the Inner Nebula}

Grain surface chemistry in the inner solar nebula is fundamentally different from that in the outer solar nebula. In the outer nebula, refractory grains serve as a substrate on which volatile species such as $\mathrm{H}_{2} \mathrm{O}, \mathrm{CO}, \mathrm{CO}_{2}, \mathrm{CH}_{4}, \mathrm{NH}_{3}$ and $\mathrm{N}_{2}$ may condense as ices; reactions may occur in the icy mantles, but there is little or no chemical interaction between the volatiles and the refractory core. In the inner nebula a dynamic chemical interplay exists between the gas and dust. "Gas-grain chemistry" there is characterized by surface-catalyzed reactions (enhancing the production rate of a volatile species over its gas-phase formation rate) and the destruction of the grains either by evaporation or by reaction with gas-phase species (enriching the nebular gas with the more refractory elements). Such reactions are highly temperature dependent, particularly at inner nebula temperatures $(\gtrsim 300 \mathrm{~K})$.

One of the most important examples of grain surface chemistry which may occur in the warm inner nebula is Fischer-Tropsch catalysis, the iron-catalyzed conversion of $\mathrm{CO}$ to $\mathrm{CH}_{4}$, a small amount of simple hydrocarbons (e.g. $\mathrm{C}_{2} \mathrm{H}_{4}$, $\mathrm{C}_{2} \mathrm{H}_{6}, \mathrm{C}_{3} \mathrm{H}_{6}, \mathrm{C}_{3} \mathrm{H}_{8}$ ), and a refractory carbonaceous residue on the catalyst surface. This process is most efficient at $400 \mathrm{~K} \lesssim T \lesssim 800 \mathrm{~K}$, and thus is the only available thermally-driven route to access the carbon locked up in $\mathrm{CO}$ in the solar nebula, because the strength of the $\mathrm{CO}$ bond precludes it from participating in gas-phase reactions under nebular conditions. While Fischer-Tropsch catalysis is capable of forming complex organic compounds under optimized industrial conditions, in the nebula the dominant product of the reaction between $\mathrm{CO}$ and $\mathrm{H}_{2}$ on $\mathrm{FeNi}$ grains will be $\mathrm{CH}_{4}(>90 \%)$, with heavier hydrocarbons becoming progressively more trace (Llorca \& Casanova 1998). Therefore, Fischer-Tropsch catalysis does not appear to be directly accountable for the diversity of organics found in carbonaceous chondrites, but it may have allowed access to the large reservoir of carbon bound as $\mathrm{CO}$, forming the precursors to meteoritic compounds.

While other materials have been suggested to catalyze this reaction in the nebula, the only viable catalyst material present there would have been metallic FeNi alloy grains, which are ubiquitous in the fine-grained matrix of chondrites (Nagahara 1984) and may originate in the nebular gas (Meibom et al. 1999). FeNi grains have also been isolated from chondritic porous interplanetary dust particles (IDPs) and were found to be embedded in and coated with carbonaceous material reminiscent of that found on industrial catalysts, suggesting that a process similar to Fischer-Tropsch catalysis occurred in the nebula (Bradley et al. 1984). Brearley (1990) finds a ubiquitous association between FeNi metal 


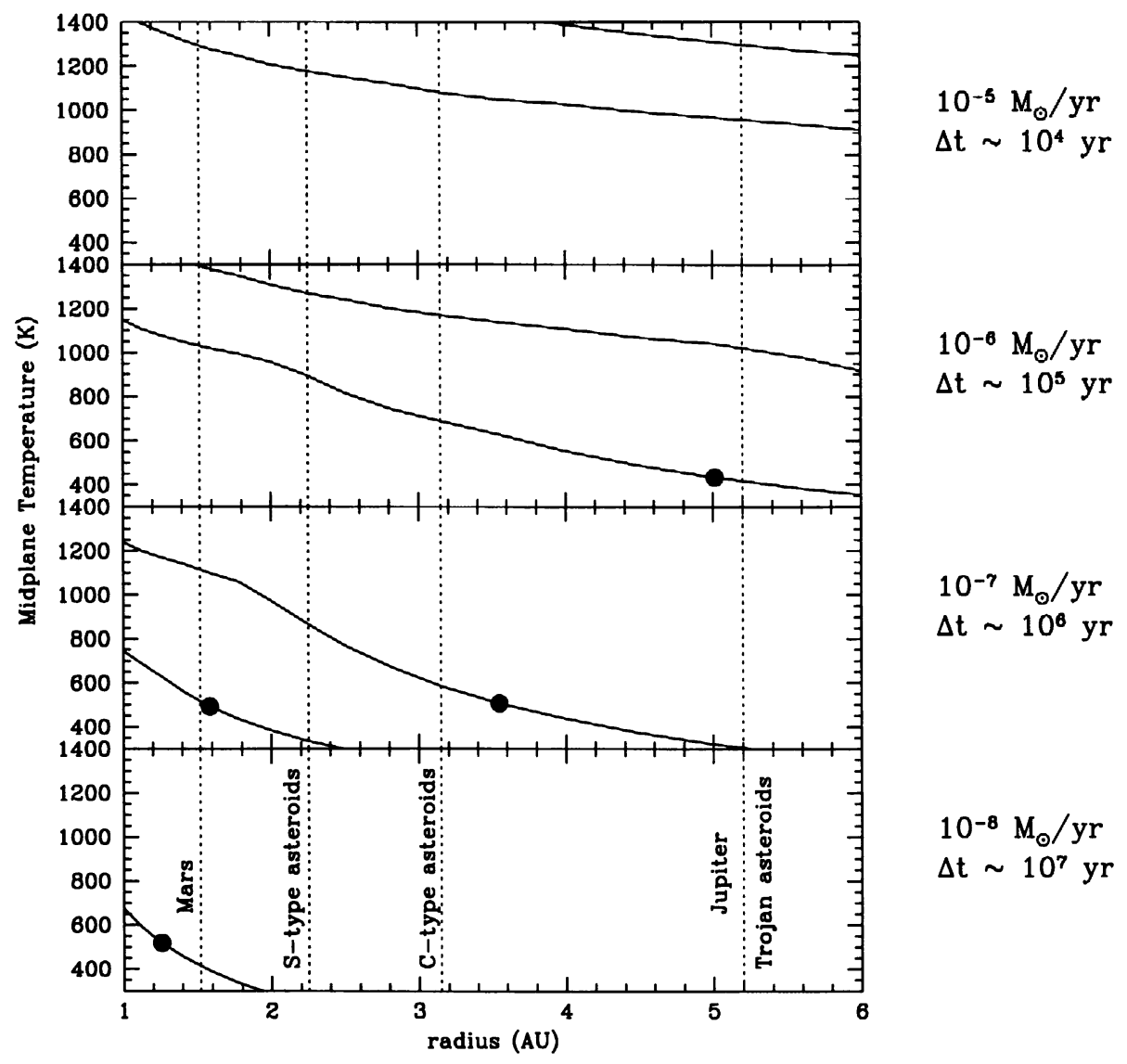

Figure 1. The range of disk midplane temperatures $\left(T_{\text {mid }}\right)$ in which meteorites formed, as a function of position in the solar nebula (data from Bell et al. 1997). $T_{\text {mid }}(r)$ evolves over time since it depends on the mass accretion rate $\dot{M}$ (labeled on the figure along with the timescale over which it persists for a solar-mass star). The viscosity parameter $\alpha$ ranges from $10^{-4}$ to $10^{-2}$, setting upper and lower limits to $T_{\text {mid }}$, respectively. The range of $T_{\text {mid }}$ shown here is from $400 \mathrm{~K}$ (the meteorite agglomeration temperature) to $1400 \mathrm{~K}$ (the silicate vaporization temperature). Conditions were suitable for condensation and agglomeration when $\dot{M}=10^{-6}-10^{-7} \mathrm{M}_{\odot} /$ year. The filled circles indicate where in the nebula the maximum rate of $\mathrm{CH}_{4}$ formation via hydrogenation of $\mathrm{CO}$ on $\mathrm{Fe}$ grains would have occurred. This process, and thus the formation of carbon-rich aggregates, would have been most efficient during the time and at the position that the meteorites and asteroids formed (figure adapted from Figure 3 of Bell et al. 2000). 
grains and carbonaceous material in carbon-rich aggregates from several ordinary chondrites. This association is highly unlikely to be coincidental, and these assemblages are believed to be physical evidence of $\mathrm{CO}$ dissociating on the surfaces of $\mathrm{FeNi}$ grains in the nebula. Because this reaction takes place in a fairly narrow but well-defined temperature range, such assemblages can place constraints on the nebular conditions during the epoch of meteorite and IDP formation, and also on the amount of $\mathrm{CO}$ which could have been converted to simple hydrocarbons.

Prinn \& Fegley (1989) investigated Fischer-Tropsch catalysis as a possible route to form $\mathrm{CH}_{4}$ in the solar nebula. They point out that the iron grains in the nebula were likely "poisoned" by the buildup of carbon on their surfaces. Experiments have shown that, on an iron catalyst, poisoning is more pronounced at higher temperatures, reducing the conversion efficiency of $\mathrm{CO}$ to $\mathrm{CH}_{4}$ (e.g. Krebs et al. 1979). These experiments also show that the buildup of carbonaceous residues occurs under the same conditions in which $\mathrm{CO}$ to $\mathrm{CH}_{4}$ conversion is most efficient, because a critical step in this process is the dissociation of the $\mathrm{CO}$ molecule.

To determine the efficiency of iron-catalyzed $\mathrm{CO}$ conversion to $\mathrm{CH}_{4}$ in the solar nebula, Kress \& Tielens (1999) have developed a kinetic model for this process which includes the effects of poisoning. Disk midplane temperature and density profiles calculated by Bell et al. (1997, see Figure 1) are used to constrain when and where in the solar nebula this process occurred. The filled circles in Figure 1 denote the position at which the maximum in the CO-to$\mathrm{CH}_{4}$ conversion rate occurs for each model, identifying the time and place in the solar nebula at which the carbon-rich aggregates and carbon-coated FeNi IDPs may form. Conditions are optimal for meteorite formation during the time when $\dot{M}=10^{-6}$ to $10^{-7} \mathrm{M}_{\odot} /$ year, because the midplane temperatures are cool enough to allow minerals to condense, and the timescales are long enough to allow the coagulation of meter-sized bodies from nebular dust. Therefore, $\mathrm{CO}$ dissociation on $\mathrm{FeNi}$ grains (and conversion to $\mathrm{CH}_{4}$ and the formation of the carbon-rich aggregates and IDPs) would have been most efficient during the time in which the meteorites and asteroids formed. At earlier times (when the mass accretion rate was very high, $\sim 10^{-5} \mathrm{M}_{\odot}$ per year), nebular conditions may have been suitable for FeNi-catalyzed dissociation of $\mathrm{CO}$ as far out as $30 \mathrm{AU}$. However, the timescale over which these conditions persisted were very short. The window of opportunity for forming $\mathrm{CH}_{4}$ and the carbon-rich aggregates moves in through the nebula as the mass flux decreases and the disk cools. This window closes completely after a few million years.

In Figure 2, the $\mathrm{CH}_{4}$ formation rate calculated using the model of Kress \& Tielens (1999) is compared to that calculated using an empirical equation derived from experimental data (Vannice 1975) for the nebula model of Bell et al. (1997) which best represents the solar nebula during the onset of meteorite and asteroid formation $\left(\dot{M}=10^{-6} \mathrm{M}_{\odot} /\right.$ year and $\left.\alpha=10^{-2}\right)$. These rates are compared to the $\mathrm{CH}_{4}$ to total carbon ratio expected at thermodynamic equilibrium (Gordon \& McBride 1971). Since the rates depend on the amount of catalyst surface area, we do not attempt to quantify the exact conversion rate which operated in the nebula or to scale the two methods of calculating the rate. These results illustrate the importance of including the effects of poisoning when 


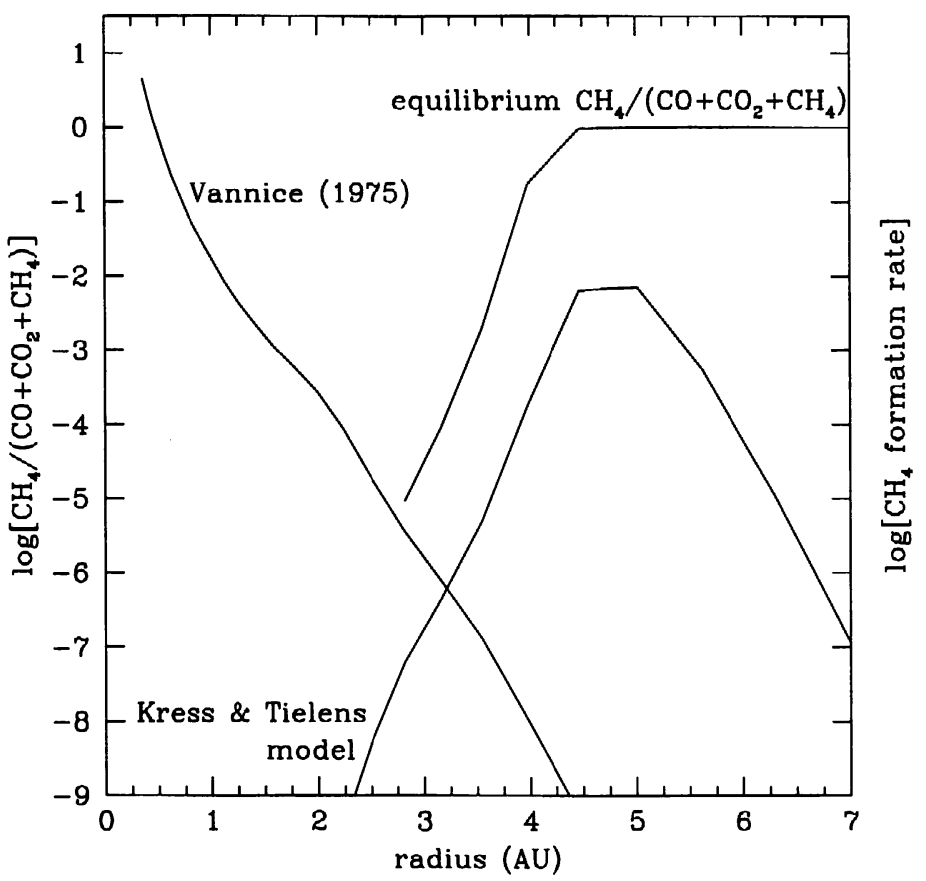

Figure 2. The $\mathrm{CH}_{4}$ to total carbon ratio at thermodynamic equilibrium as a function of position in a protoplanetary disk at the onset of the meteorite-forming epoch $\left(\dot{M}=10^{-6} \mathrm{M}_{\odot} /\right.$ year and $\left.\alpha=10^{-2}\right)$. Shown on the same relative scale is the $\mathrm{CH}_{4}$ formation rate calculated using an empirical equation derived from rate data taken at high pressures (Vannice 1975) and the model developed by Kress \& Tielens (1999), which includes poisoning of the catalyst grains.

calculating the $\mathrm{CH}_{4}$ formation rate in the solar nebula. Kress \& Tielens (1999) show that poisoning is quite rapid, so that the $\mathrm{CO}$ to $\mathrm{CH}_{4}$ conversion rate in the nebula is not a monotonically increasing function of temperature, as the Vannice equation suggests. Instead, in warmer regions where the thermodynamically favored form of carbon is $\mathrm{CO}$, the $\mathrm{CH}_{4}$ formation rate is low. The rate increases outward through the nebula, in excellent agreement with the trend for $\mathrm{CH}_{4}$ to become a more stable nebular species at progressively larger radii (cooler temperatures). At very large heliocentric distances, the midplane temperature drops below that sufficient for $\mathrm{CO}$ to dissociate on iron grains, and the conversion rate drops due to kinetic inhibition. On the other hand, the Vannice equation blindly predicts that the $\mathrm{CO}$ to $\mathrm{CH}_{4}$ conversion rate is highest in the hot inner nebula and decreases monotonically with the disk midplane temperature. This comparison illustrates the fact emphasized by Kress \& Tielens (1999) that empirical equations taken from the chemical engineering literature (such as Vannice 1975) cannot be reliably or meaningfully extrapolated to astrophysical conditions. 


\section{Conclusions and Future Outlook}

In order to determine the history of the inner solar nebula, we must first understand the physical conditions experienced by interstellar material prior to and during its incorporation into planetesimals. The chemical and physical evolution of the warm inner region of disks are intimately dependent on one another; therefore, the solar nebula must be treated as a time-dependent process, not as a static object. Theoretical models for the evolution of accretion disks and the physical phenomena within them provide a framework within which to study the effects of grain-surface, gas-phase, thermally-driven, photochemical and ionmolecule reactions in the terrestrial-planet-forming regions of protoplanetary nebulae.

$\mathrm{CO}$ dissociation and hydrogenation on FeNi grains in the solar nebula is one example of the dynamic chemistry expected between the gases and grains of the inner nebula. Laboratory work is essential to develop realistic models for this and many other gas-solid reactions which are expected to occur in the solar nebula. These models may then be incorporated into kinetic models for solar nebula chemistry. A more detailed understanding of inner nebula chemistry will help constrain current models for planetesimal formation and enable us to fill in the gaps in our current knowledge of the link between meteoritic data and observations of protoplanetary disks.

With current instrumentation, we are rather far from being able to determine chemical abundances in the inner regions of protoplanetary disks. Some of the most exciting prospects for studying inner solar nebula chemistry from an astrophysical perspective is the development of large millimeter and submillimeter arrays, such as the Sub-Millimeter Array (SMA) and the Atacama Large Millimeter Array (ALMA). With sufficiently high resolution, large chemical abundance gradients between the inner and outer portions of protoplanetary disks will be detectable, providing the first glimpse of the chemistry of the terrestrial-planet-forming region of solar nebula-like objects.

Acknowledgments. The author made extensive use of NASA's Astrophysics Data System Abstract Service to search the very diverse body of literature relevant to inner solar nebula studies. She is grateful to K.R. Bell for providing the disk temperature and density profiles used in this work, S. Desch for enlightening discussions about lightning, and $\mathrm{H}$. Connolly, A. Boogert, D. Backman, D. Carbon, F. van der Tak, M. Bernstein, and J. Chambers for their insightful comments which helped to improve this paper.

\section{References}

Aikawa, Y., Umebayashi, T., Nakano, T., \& Miyama, S.M. 1999, ApJ, 519, 705 Bell, J.F., Davis, D.R., Hartmann, W.K., \& Gaffey, M.J. 1989, in Asteroids II, eds. R.P. Binzel, T. Gehrels, \& M.S. Matthews (Univ. of Arizona Press: Tucson), 921

Bell, K.R., Cassen, P.M., Klahr, H.H., \& Henning, Th. 1997, ApJ, 486, 372

Bell, K.R., Cassen, P.M., Wasson, J.T., \& Woolum, D.S. 2000, in Protostars and Planets IV, eds. V. Mannings, A. Boss, \& S. Russell (Univ. of Arizona Press: Tucson) 
Borucki, W.J. \& McKay, C.P. 1987, Nature, 328, 509

Boss, A.P. 1993, ApJ, 417, 351

1996, in Chondrules and the Protoplanetary Disk, eds. R.H. Hewins,

R.H. Jones, \& E.R.D. Scott (Univ. Press: Cambridge), 257

Bradley, J.P., Brownlee, D.E., \& Fraundorf, P. 1984, Science, 223, 56

Brearley, A.J. 1990, Geochim. Cosmochim. Acta, 54, 831

Cassen, P.M. 1996, Meteorit. Planet. Sci., 31, 793

Desch, S.J. \& Cuzzi, J.N. 2000, Icarus, in press

Duschl, W.J., Gail, H.-P., \& Tscharnuter W.M. 1996, A\&A, 312, 624

Dutrey, A., Guilloteau, S., \& Guélin, M. 1997, A\&A, 317, L55

2000, this volume

Fegley B. 1993, in Chemistry of Life's Origins, eds. J.M. Greenberg, C.X. Mendoza-Gómez, \& V. Pirronello (Kluwer Acad. Pub.: Dordrecht), 75

Finocchi, F., Gail, H.-P., \& Duschl, W.J. 1997, A\&A, 325, 1264

Finocchi, F. \& Gail, H.-P. 1997, A\&A, 327, 825

Gail, H.-P. 1998, A\&A, 332, 1099

Gordon, S. \& McBride B. 1971, NASA Special Publications SP-273

Horanyi, M., Morfill, G., Goertz, C.K., \& Levy, E.H. 1995, Icarus, 114, 174

Igea, J. \& Glassgold A.E. 1999, ApJ, 518, 848

Krebs, H.J., Bonzel, H.P., \& Gafner, G. 1979, Surf. Sci., 88, 269

Kress, M.E. \& Tielens, A.G.G.M. 1999, Meteorit. Planet. Sci., submitted

Lewis, J.S. 1974, Science, 186, 440

Llorca, J. \& Casanova, I. 1998, Meteorit. Planet. Sci., 33, 243

McKay, C.P. \& Borucki, W.J. 1997, Science, 276, 390

Meibom A. \& Clark, B.E. 1999, Meteorit. Planet. Sci., 34, 7

Meibom, A., Petaev, M.I., Krot, A.N., \& Wood, J.A. 1999, J. Geophys. Res., 104,22053

Nagahara, H. 1984, Geochim. Cosmochim. Acta 48, 2581

Podolak, M. \& Bar-Nun, A. 1988, Icarus, 75, 566

Prinn R. \& Fegley B. 1989, in Origin and Evolution of Planetary and Satellite Atmospheres, eds. S.K. Atreya, J.B. Pollack, \& M.S. Matthews (Univ. of Arizona Press: Tucson), 78

Qi, C., Blake, G.A., \& Sargent, A.I. 1999, IAU Symposium 197, poster book, 228

Stepinski, T.F. 1998, Icarus, 132, 100

Thi, W.-F., van Zadelhoff, G.J., van Dishoeck, E.F., Qi, C., \& Blake, G.A. 1999, IAU Symposium 197, poster book, 233

Vannice, M.A. 1975, J. Catal., 37, 449

Velusamy, T., Langer, W.D., \& Goldsmith, P.F. 1999, IAU Symposium 197, poster

Wetherill, G.W. \& Chapman, C.R. 1988, in Meteorites and the Early Solar System, eds. J.F. Kerridge \& M.S. Matthews (Univ. of Arizona Press: Tucson), 35

Willacy, K., Klahr, K.K., Millar, T.J., \& Henning, Th. 1999, A\&A, 338, 995

Woolum, D.S. \& Cassen, P. 1999, Meteorit. Planet. Sci., 34, 897 


\section{Discussion}

D. Johnstone: Yesterday we heard that the "snowline" might not be important and that the location of ices might not be dependent on this. How does this fit in with your discussions today?

M. Kress: I only mentioned the snowline in the sense that it distinguishes the "inner" nebula from the "outer" as where ices can exist and where they cannot.

P. Caselli: Could you please explain to me how the $\mathrm{CO}$ molecule gets dissociated onto the grain surface by the Fischer-Tropsch catalysis process? Is there any possibility for $\mathrm{C}$ and $\mathrm{O}$ to recombine in $\mathrm{CO}$ ?

$M$. Kress: If the $\mathrm{O}$ is not removed as $\mathrm{H}_{2} \mathrm{O}$ or $\mathrm{CO}_{2}$, the $\mathrm{C}$ and $\mathrm{O}$ may recombine. But this does not happen often enough to preclude the formation of the carbide adlayer. Transition metals have the unique property that their $d$ electrons interact with both the bonding and antibonding orbitals of $\mathrm{CO}$, binding it to the surface, but then breaking the $\mathrm{C}=\mathrm{O}$ bond.

$J . M$. Greenberg: I wonder if you could estimate what proportion of "gloop"coated metal grains might be found in comets? I appreciate the possibility of their contribution to meteorites but even then is there a problem with sizes? You showed a $0.1 \mu \mathrm{m}$ example but in meteorites the particles are much larger.

M. Kress: If coated grains are found in comets, it may suggest the coatings formed outside the solar system since they require high temperature to form. The fraction of coated iron grains is hard to estimate but would serve as constraints on the amount of iron which participated in this reaction.

Y. Aikawa: You express the reaction efficiency in "site ${ }^{-1} \mathrm{sec}^{-1}$." Do you have any estimate how many sites there are on the grain surface?

$M$. Kress: Meteoritic and IDP data will constrain how much surface area is available for reaction. The model results suggest that the rate is efficient enough to have an effect on the gas, without requiring more than a few $\%$ of the iron in metallic grains, in reasonably-sized particles $(\sim 1 \mu \mathrm{m}$ to $10 \mu \mathrm{m})$.

$F$. Bensch: Which (other) evidences are there in favor of the lightning theory?

M. Kress: I would refer you to Steve Desch's paper which was presented at Protostars and Planets IV, and will appear in Icarus (Desch \& Cuzzi 2000). Whatever melted the chondrules had to be rapid and repeatable, and so far lightning is the only melting mechanism that satisfies these criteria.

$W$. Irvine: How far out in the nebula might lightning play an important chemical role?

M. Kress: Lightning via triboelectric charging of grains requires different grain types, so if these are covered in ice, this may not work. Also, fairly high density neutral gas is required, which suggests lightning may occur out to about $5 \mathrm{AU}$. Refer to Desch \& Cuzzi (Icarus 2000). 